vol. $27-n^{\circ} 3 \mid 2011$

Numéro ouvert

\title{
Histoire du développement de la recherche universitaire française sur les migrations internationales (1815-1999)
}

History of French Academic Researches' Development on International Migrations (1815-1999)

Historia del desarrollo de la investigación de las universidades francesas de las migraciones internacionales (1815-1999)

\section{Latifa Benabou-Lucido}

\section{OpenEdition}

Journals

Édition électronique

URL : https://journals.openedition.org/remi/5609

DOI : 10.4000/remi.5609

ISSN : $1777-5418$

Éditeur

Université de Poitiers

Édition imprimée

Date de publication : 1 décembre 2011

Pagination : 7-30

ISBN : 979-10-90426-02-3

ISSN : 0765-0752

\section{Référence électronique}

Latifa Benabou-Lucido, « Histoire du développement de la recherche universitaire française sur les migrations internationales (1815-1999) », Revue européenne des migrations internationales [En ligne], vol. $27-n^{\circ} 3$ | 2011, mis en ligne le 01 décembre 2014, consulté le 14 avril 2022. URL : http:// journals.openedition.org/remi/5609; DOI : https://doi.org/10.4000/remi.5609 


\title{
Histoire du développement de la recherche universitaire française sur les migrations internationales (1815-1999)
}

\section{Latifa BENABOU-LUCIDO ${ }^{1}$}

\begin{abstract}
«La recherche interdisciplinaire n'existe que parce qu'il existe des disciplines... ! Si l'on ajoute à cette évidence une autre évidence à savoir que la recherche interdisciplinaire est considérée aujourd'hui comme une démarche intellectuelle qui doit être favorisée, on en vient naturellement à s'interroger sur les fondements de la division du savoir en disciplines » (Reisse, 1999 : 13).
\end{abstract}

$M$ on propos ici, n'est pas de discuter l'importance évidente de la recherche interdisciplinaire en matière d'étude des migrations, mais de souligner l'éclatement de celle-ci entre les différentes disciplines de la recherche française.

Pour examiner le statut des migrations dans la recherche française, tenter de montrer les logiques des travaux universitaires sur les migrations, suivre donc la progression de la problématique migratoire dans les différentes disciplines, la question des sources à utiliser s'est posée d'emblée. Quel support utiliser ? Les articles des revues scientifiques spécialisées, les colloques, les thèses, les mémoires des Masters I et II, les ouvrages édités ou tous ces supports à la fois ? Cette dernière solution exhaustive aurait été bien évidemment la plus intéressante.

Le statut des migrations dans la recherche française n'ayant constitué qu'un chapitre de ma thèse, il était nécessaire de délimiter le champ de ma recherche. J'ai donc choisi le seul support constitué par les thèses de troisième cycle, soutenues en France.

1 Responsable pédagogique du SCFC, Université Paris Descartes, 45 rue des Saints-Pères, 75006 Paris ; latifa.benabou-lucido@parisdescartes.fr 
L'état des lieux a porté au final sur l'analyse de 1882 thèses ${ }^{2}$ sur les migrations, classées chronologiquement, réparties par décennie et couvrant une période allant de $1815^{3}$ à $1999^{4}$. Il met en évidence la progression de la problématique des migrations dans les différentes disciplines de la recherche française ${ }^{5}$. Il montre qu'au cours de la période examinée, les disciplines qui ont étudié les migrations sont en majorité, le Droit $^{6}(308)^{7}$ et la Médecine (288), suivies par la Sociologie (251), la Géographie (188) et l'Histoire (121). Les 659 thèses restantes sont partagées entre le reste des disciplines ${ }^{8}$.

Jusqu'aux années 1960, l'étude des migrations a été largement menée par le Droit et ce n'est qu'à partir de ces années qu'un renouvellement dans l'étude des migrations est apparu tant au niveau des problématiques traitées, des disciplines qui s'y sont intéressées qu'au niveau des populations étudiées. En effet, en plus du Droit, de la Médecine (4) et des Lettres (15), de nouvelles ${ }^{9}$ disciplines comme la Géographie, les Sciences économiques, la Sociologie, l'Histoire, etc. tentent de développer leurs recherches dans ce domaine et certaines y montrent un intérêt grandissant. L'entrée tardive de ces disciplines qui existaient de facto avant ces années, interroge cependant et l'on peut supposer qu'elles étaient confrontées à de « puissantes résistances à l'innovation » (Mazon, 1988:11) dans l'Université française, auprès de la communauté scientifique, littéraire et juridique.

Le renouvellement des problématiques traitées trouve son explication dans l'effervescence qu'a connue l'Université française à la fin des années 1960, sous l'impulsion d'institutions anciennes comme l'École pratique des Hautes Études ou d'autres ${ }^{10}$ qui sont créées pendant ces années et par conséquent, dans le déplacement des problèmes sociaux à l'intérieur de l'Université et de la recherche. Pendant ces années, on s'intéresse, notamment, aux relations entre syndicats et travailleurs immigrés, aux migrations de loisirs, ou encore aux aspirations culturelles des immigrés. Cette approche est inédite, car pour la première fois, l'on s'intéresse aux immigrés en empruntant une perspective qui dépasse leur seule fonction de travailleurs ou d'ouvriers.

2 Cf. annexe 1. Recensement et analyse des thèses réalisés entre 1999 et 2005, réactualisés en décembre 2009.

3 Date de la première thèse recensée par le Système universitaire de documentation (Sudoc) au cours de ma recherche (1999-2005 et 2009). Depuis, des thèses antérieures à celle de 1815 ont été probablement ajoutées dans cette base de données.

4 J'ai procédé à une analyse des thèses par décennie. Dès lors, toutes les thèses soutenues à partir de 2000 ont été écartées.

5 Pour l'inventaire des thèses et les références des exemples fournis dans cet article, cf. BenabouLucido, 2008 : annexe 9.

6 Le Droit, non pas sous sa forme actuelle d'une science juridique, mais depuis les origines de l'université de Paris (XIIIe siècle), occupait une place parmi les sept arts libéraux (grammaire, didactique et rhétorique, arithmétique, musique, géométrie et astronomie, théologie, droit canon savoirs cléricaux - et médecine) qui constituaient alors toute la sagesse humaine.

7 Entre parenthèses : total des thèses.

8 Cf. annexe 2.

9 Cet adjectif est utilisé ici dans le sens de l'intérêt de la discipline à l'étude des migrations.

10 Comme la Faculté des Lettres de Nanterre, créée en 1964. 


\section{DES ARTS LIBÉRAUX POUR ÉTUDIER LES MIGRATIONS}

\section{Le Droit}

La recherche universitaire sur les migrations en Droit a commencé dès le début du XIXe siècle et se prolonge encore au cours du XXIe siècle, ce qui explique l'importance quantitative des thèses qui y sont soutenues. L'une des raisons de cette importance quantitative est l'apparition d'un nouveau domaine portant sur la politique, l'économie et la société entre 1865 et 1919. Les facultés de Droit voulant s'imposer en matière d'enseignement de ce que nous appellerons par la suite les « Sciences sociales », des chaires d'économie politique, de législation comparée, d'économie coloniale, de droit institutionnel s’y sont multipliées.

La recherche universitaire sur les migrations s'est consacrée surtout à l'analyse des textes juridiques concernant d'abord les étrangers (1815-1899), ensuite les différentes catégories d'immigrés (1900-1939) et enfin, et dans une moindre mesure, leur descendance à partir des années 1980. Louis Liard (1846-1917), directeur de l'enseignement supérieur en 1884, considérait que les facultés de Droit étaient « closes de toutes parts, sauf du côté du barreau et de la magistrature » (Liard, 1890 : 80). Les thèses soutenues se sont attachées en effet à interpréter la loi écrite et à examiner son application aux étrangers sans interroger les faits qui pourraient la modifier, ni introduire des doutes par rapport à son immuabilité supposée. Henri Lévy-Bruhl (1884-1964), formé à la fois au Droit et à la Sociologie, défendant l'incorporation de la Sociologie au Droit, écrira plus tard que « ils [les juristes] auraient appris au contact de cette discipline [la Sociologie], que les phénomènes sociaux ont des causes "sociales" » et que « les règles juridiques étant l'expression des groupes et non d'individus, acquièrent par là-même une objectivité qui les rend justiciables de l'investigation scientifique » (Lévy-Bruhl, 1950 : 123).

Au cours du XIXe siècle, la condition civile et légale de l'étranger en France correspondait largement à la problématique étudiée en Droit, ce que Nancy Green a montré dans ses recherches portant sur l'historiographie comparée entre la France et les ÉtatsUnis : « [...] le terme "étranger" est de loin le substantif ou l'adjectif (ouvriers étrangers, travailleurs étrangers, immigration étrangère) préféré » (Green, 1991 : 69).

$\mathrm{Au}$ milieu de ce siècle et dans le contexte de la dépression économique, de nouvelles préoccupations politiques et sociales autour de l'étranger ont favorisé de nouveaux sujets de recherche : l'asile et le droit d'expulsion, l'assimilation, l' " acquisition de la qualité de Français » ou encore les professions que les étrangers étaient autorisés à exercer.

Le terme d'étranger, au début du XXe siècle, sans disparaître de la recherche universitaire française, cède la place à celui d'ouvrier ou il lui est systématiquement accolé. Les thèses analysées étudient l'ouvrier étranger, son rôle dans l'économie, les modalités de son recrutement, son statut juridique, les moyens de réguler et de quantifier 
sa présence, d'évaluer le risque qu'il présente pour le travail national afin que l'État puisse s'en protéger. La majorité des thèses examine l'immigration ${ }^{11}$ des ouvriers en France et celle des Français à l'étranger et en France. Cette classe ouvrière a suscité l'étude de questions comme la protection du travail national, les accidents du travail, les lois d'assurance et de responsabilité, etc. Au cours de ces années, l'étranger est mieux identifié ; il est italien ou asiatique. On remarque également que les études deviennent plus précises, plus locales et concernent les migrations internes en France et leurs effets sur la propriété territoriale, ou leurs conséquences économiques, sociales et urbaines. Pendant cette période, l'émigration française est étudiée également au Mexique ou au Maroc ainsi que dans les colonies ou dans d'autres pays étrangers.

La propension à l'identification des étrangers se poursuit après la Première Guerre mondiale où la recherche universitaire délimite davantage les populations étudiées (Polonais, Algériens, Marocains, Russes) et les régions dans lesquelles elles sont implantées. Ces orientations persistent pendant les années 1920 et jusqu'aux années 1940.

Entre 1940 et 1959, période marquée par la Deuxième Guerre mondiale, on note une chute du total des thèses dans l'ensemble des disciplines qui ont porté sur les migrations $^{12}$. Si on excepte la population réfugiée et apatride, on ne constate pas de renouvellement significatif ni des populations, ni des sujets étudiés. L'étude des migrations suit les mêmes axes dégagés plus haut.

Néanmoins, au cours des années 1970, les thèses en Droit adoptent, pour certaines, une perspective plus sociale en étudiant, par exemple, la condition sociale du travailleur immigré algérien en France. Elle intègre une dimension comparative avec l'étude des migrations rurales dans la Communauté économique européenne, ou encore les politiques comparées de l'immigration en France et en Grande-Bretagne.

Les préoccupations premières de la recherche juridique (expulsion et extradition des étrangers, statut juridique) persistent, au cours des années 1980 et 1990, bien que les termes (mesures d'éloignement au lieu d'expulsion) changent durant les années 1990. On assiste, cependant, à un glissement vers le « culturel ${ }^{13}$ qui va perdurer pendant cette même décennie. Pour la première fois, on considère les représentations de l'immigré et leurs effets sur les comportements sociaux. De nouvelles problématiques se sont construites autour de l'Islam ${ }^{14}$, de l'étranger en situation irrégulière, ou de l'étranger incarcéré. La démarche comparative avec d'autres contextes sociaux se développe aussi au cours de ces années.

11 Le terme d'immigré n'était pas encore utilisé.

12 Une recherche rapide afin de vérifier si cette chute est générale, si elle concerne les autres sujets de la recherche, permet de constater que ceux-ci ont été touchés différemment. J'ai choisi au hasard six mots-clés (guerre(s), paix, nationalisme(s), religion(s), sécurité(s), travail) à partir desquels j'ai fait une recherche des thèses au cours des mêmes périodes que pour le sujet « migrations ». Les thèses autour des problématiques de la religion et de la sécurité ont progressé alors que celles autour des problématiques du travail, du nationalisme, de la paix et de la guerre ont marqué une diminution entre 1940 et 1959, sans toutefois chuter.

13 La relation entre l'identité culturelle, la condition juridique et sociale et l'intégration chez des « Maghrébins » en France.

$14 \mathrm{La}$ répudiation islamique. 


\section{La Médecine}

Bien qu'elle ait été autonome institutionnellement très tôt, la Médecine ne s'est intéressée réellement à l'étude des migrations qu'au cours des années 1980 pour traiter de manière périphérique le processus migratoire.

Avant l'explosion des années $1980^{15}$, une dizaine de thèses seulement a été enregistrée. La première thèse recensée date de 1908 et concerne la dépopulation du milieu rural. Néanmoins, on constate que les premières recherches en Médecine ont adopté une approche aussi bien historique ${ }^{16}$ que juridique ${ }^{17}$.

L'irruption des sujets concernant les migrations en Médecine atteste de son intérêt à cette problématique. Toutefois, l'examen des thèses montre que dans la majorité des recherches, l'immigration est considérée davantage comme un facteur ou une variable dans le sens de la statistique descriptive. Ce n'est pas l'immigration en tant que processus ${ }^{18}$ qui intéresse la recherche médicale, mais ce que le facteur « immigration » apporte à l'explication de certaines pathologies. Ainsi, plusieurs thèses ont concerné des populations immigrées ${ }^{19}$ en France et leurs pathologies, leur alimentation et leur éducation sanitaire, leur santé au travail, etc.

Un second axe de la recherche s'articule autour de la pratique médicale, à travers l'étude des problèmes posés au médecin par certaines populations immigrées ${ }^{20}$ ou dans des centres de soins ${ }^{21}$ ou encore dans des camps de réfugiés ${ }^{22}$ ou au sein de l'association Médecins Sans Frontières.

Cependant, un léger changement est introduit dans les orientations des recherches effectuées à partir de 1990. En effet, au lieu de s'intéresser à la population immigrée par rapport à sa pathologie physique et psychique uniquement, les problématiques ${ }^{23}$ introduisent des préoccupations plus sociales dont le but est l'ajustement des prises en charge en fonction des différentes populations.

Au cours de ces années, on note une profusion d'appellations des populations étudiées. Ainsi, les jeunes Français issus de l'immigration nord-africaine sont tantôt désignés comme les « Maghrébins de seconde génération », les " jeunes adultes nés de

15185 thèses sur les migrations soutenues en Médecine sur un total de 664 (toutes disciplines confondues) recensées entre 1980 et 1989.

16 M. Lerolle, L'exode d'un peuple : étude des conséquences sanitaires du départ des Grecs d'AsieMineure après la guerre gréco-turque de 1922, Université de Paris, 1931.

17 J. Pécout, L'étude et l'exercice de la médecine par les étrangers, Université de Paris, 1939.

18 Trois thèses seulement ont étudié les migrations dans le sens d'un déplacement d'une population d'un pays d'origine vers un pays d'accueil.

19 « Noirs africains immigrés », « enfants de parents migrants d'Afrique noire », « malades étrangers », etc.

20 Le solliciteur d'asile par exemple.

21 Le Comede par exemple.

22 En Asie, Afrique, etc. Cet axe de la recherche est poursuivi pendant les années 1990.

23 État de santé et conditions de travail des immigrés, adaptation du cadre thérapeutique en ethnopsychiatrie et amélioration de l'action médicale envers les « étrangers », etc. 
parents immigrés », tantôt comme les « adolescents maghrébins dits de deuxième génération », ou encore les « adolescents issus de migrants, deuxième génération de migrants ». Ces appellations semblent correspondre à une mise à distance, une différenciation de ces Français qui du reste ne seraient pas comme des Français de souche. Cet intérêt pour la « seconde génération » s’est développé pendant les années 1990.

Les descendants des immigrés intéressent la Médecine en tant que groupe dont tous les membres auraient des caractéristiques communes. À titre d'exemple, cette discipline examine les troubles de l'identité que présentent des adolescents issus de l'immigration, les anomalies dans le comportement scolaire d'enfants d'origine immigrée, ou l'incidence du statut d'enfants d'immigrés sur l'éthiopathogénie de la schizophrénie. Jusque-là, il ne s'agit que de l'étude de problèmes chez cette population. Cet exercice de normalisation des populations est poursuivi et l'on cherche une caractérisation identitaire de cette population ou encore l'existence d'une spécificité pathologique chez elle.

\section{LA PERSPECTIVE ÉCONOMIQUE DE L'ÉTUDE DES MIGRA- TIONS}

Dès les années 1950, la discipline Sciences économiques (3) s'est intéressée à l'étude des migrations. Au cours des années 1970, on constate un élargissement du champ d'étude des migrations, d'où l'augmentation du total des thèses (15). En plus des facteurs économiques liés aux migrations, on y étudie les problèmes de l'émigration algérienne en France, mais également son histoire. Cette approche n'est pas spécifique aux Sciences économiques, car d'autres disciplines comme la Sociologie notamment, bien qu'elles aient des champs d'étude définis, les ont souvent débordés. Durant les décennies 1980-1989 (49) et 1990-1999 (30), cette discipline oriente principalement ses recherches vers l'analyse économique des migrations, leur impact économique et social sur les pays d'origine, surtout pendant les années 1990, et vers la relation entre migration et développement économique donc sur l'étude basée sur la théorie de la production et de la circulation des biens et des hommes. De nouvelles populations sont étudiées : les femmes et leur rôle dans l'insertion mondiale des systèmes productifs français et britannique, les Tunisiens, les Marocains, les étudiants, etc.

\section{L'INSCRIPTION DES THÈSES SUR LES MIGRATIONS EN LETTRES}

Seules cinquante-sept thèses sur les migrations ont été recensées en Lettres entre 1815 et 1999 .

$\mathrm{Au}$ cours de la période 1815 à 1899 , la première thèse ${ }^{24}$ sur les migrations fut soutenue à l'université de Paris, en 1883. La seconde sera soutenue en 1923. Il faut souligner néanmoins que les thèses qui ont suivi étudient l'immigration de petits groupes,

24 J. Loth, L'émigration bretonne en Armorique : du Ve au VIIe siècle de notre ère.

REMI 2011 (27) 3 pp. 7-30 
d'un point de vue historique ${ }^{25}$, géographique ${ }^{26}$ ou sociologique ${ }^{27}$. Elles ont porté aussi bien sur l'immigration en France que sur les migrations dans d'autres pays. Ces thèses auraient eu leur place en Histoire, en 9, ou encore en Sociologie si ces disciplines avaient eu une assise institutionnelle. La thèse de Georges Mauco, qui est une thèse de géographie, soutenue en Lettres en 1932, atteste en effet des résistances de l'Université française à faire une place aux nouvelles disciplines.

L'on constate ainsi que des thèses relevant d'autres disciplines (comme l'Histoire $^{28}$, par exemple), au cours des années 1940 et jusqu'à la fin des années 1950, ont été toujours soutenues en Lettres. Durant cette période, on assiste, néanmoins, à une nouvelle manière d'étudier les migrations à travers l'analyse des œuvres d'écrivains ${ }^{29}$.

Dans le suivi de la genèse des disciplines des Sciences humaines et sociales, il est important d'insister sur le fait que les premières thèses soutenues dans les facultés de Lettres relevaient de disciplines telles que la Géographie, l'Histoire, etc. et seule l'absence d'un ancrage institutionnel autonome les faisait dépendre de cette faculté. Louis Liard s'interrogeait sur les « disciplines à frontières indécises, comme la Géographie, l'Histoire, la Philosophie elle-même, qu'on peut traiter tantôt comme une section des Lettres, tantôt comme un chaînon des Sciences. [...] les placer à demeure fixe, en vertu d'une organisation conventionnelle, soit à la Faculté des lettres, soit à la Faculté des sciences, n'est-ce pas les condamner à d'inévitables arrêts de développement ?» (Liard, 1890 : 145-146). Certes, mais si ces disciplines avaient eu leur autonomie institutionnelle assurée au sein de l'Université, loin des résistances et des luttes des facultés, cela aurait pu favoriser, non seulement leur autonomie scientifique, mais également la possibilité d'une interdisciplinarité pour l'étude des faits de société.

Si l'on tente de reclasser les thèses soutenues sous la houlette de la faculté des Lettres, on remarque que sur les cinquante-sept thèses soutenues en Lettres, douze au moins auraient pu relever de l'Histoire ${ }^{30}$, sept au moins de la Géographie Gl $^{31}$ et quatre au minimum de la Sociologie $e^{32}$.

On constate, entre 1970 et 1989, les mêmes orientations de la recherche universitaire en Lettres - et Sciences humaines. Les thèses soutenues étudient les migrations

25 E. Philips, Les réfugiés bonapartistes en Amérique (1815-1830), Université de Paris, 1923.

26 G. Mauco [1899-1988], Les étrangers en France. Étude géographique sur leur rôle dans l'activité économique, Université de Paris, 1932.

27 K.-H. Weng, Essai sur l'immigration chinoise en Insulinde : (étude sociologique), Université de Paris, 1937.

28 M.-V. Sakellariou, La migration grecque en Ionie, Université de Paris, 1958 ; P. Ikowswi, L'Émigration des Italiens, des Polonais et des Allemands et leur immigration en France dans la région parisienne, Université de Paris, 1952, etc.

29 R. Walch, Le tragique de l'émigration dans la poésie de Mickiewiez (1798-1855), Université de Lyon 2, 1944.

30 La thèse de J. Loth citée ci-dessus ; C.-R. Ageron, Les Algériens musulmans et la France (18711919), 1966.

31 La thèse de G. Mauco citée ci-dessus.

32 La thèse de K.-H. Weng citée ci-dessus. 
de populations en France ${ }^{33}$, les migrations internes en France et enfin, les migrations et leurs effets dans d'autres pays. Cette tendance se poursuit pendant les années 1990. Bien qu'elles relèvent de l'Histoire ${ }^{34}$, de la Géographie ${ }^{35}$ ou de la Sociologie ${ }^{36}$, des thèses continuent encore à être soutenues en Lettres et ont vu leur total augmenter (16). Le reste des thèses traite de la relation entre immigration et littérature.

\section{La sociologie}

La Sociologie fut intégrée pour la première fois à l'Université française en 1887, à Bordeaux, par le biais du cours Science sociale et pédagogie, confié à Émile Durkheim (1858-1917) et ceci, malgré les protestations des professeurs de la faculté de Droit parmi lesquels on comptait pourtant des défenseurs de la Sociologie comme Fernand Faure (1853-1929) ou Léon Duguit (1859-1928). Dès que la nomination d'Émile Durkheim à l'université de Bordeaux est rendue publique, Léon Duguit n'hésita point à réclamer l'exclusivité de l'enseignement des Sciences sociales, y compris la Sociologie, aux facultés de Droit.

Plusieurs débats et échanges d'articles entre partisans et opposants à la Sociologie ont suivi, jusqu'au positionnement d'Ernest Lavisse ${ }^{37}$ (1842-1922). Celui-ci publie au Journal des Débats, le 14 novembre 1893, un article dans lequel il défend le développement de l'enseignement des Sciences sociales. Trois jours plus tard, il publie un second article, dans le même journal, en réaction à l'achat par l'université de Montpellier d'un vignoble, où il assure que l'université de Paris pourrait avoir les moyens pour un enseignement en Sciences sociales à condition « [...] qu'on nous donne [...] de l'argent, des terres, des vignes, tout ce qu'on voudra [...]» (Weisz, 1979: 93). C'est seulement grâce à l'aide financière du Comte Aldebert de Chambrun (1872-1963) que la fondation d'une chaire d'économie sociale à la Sorbonne a été possible.

Les juristes, qui estimaient que l'enseignement de la Sociologie relevait de leur faculté, n'allaient pas tarder à manifester leurs inquiétudes. Sur le plan idéologique, ils s'estimaient plus modérés que les professeurs des facultés des Lettres. Cependant, au cours de cette année (1893), grâce à la thèse d'Émile Durkheim, à la fondation de la Revue internationale de sociologie (RIS) et à la création de l'Institut international de sociologie par René Worms (1869-1926), la Sociologie s'impose et accède à une certaine autonomie et son développement, bien que difficile, se poursuit jusqu'à l'année 1944.

Dès 1904 , le cours d'histoire et d'économie sociale avait été transformé en chaire d'histoire et d'économie sociale grâce au soutien financier du ministère de l'Instruction Publique. Émile Durkheim était alors professeur titulaire de la chaire de Science de l'édu-

33 Yougoslaves, travailleurs tunisiens.

34 L'émigration corse à Porto-Rico, l'histoire de l'émigration kabyle en France au XXe siècle.

35 L'organisation urbaine en Algérie.

36 La migration et la réussite sociale à travers l'exemple de la bourgeoisie marocaine, l'exclusion sociale aux États-Unis.

37 Historien, directeur de l'École normale supérieure (ENS) entre 1904 et 1919.

REMI 2011 (27) 3 pp. 7-30 
cation, à Paris. Célestin Bouglé38 (1870-1940) obtint, en 1919, la chaire d'Histoire d'économie sociale qui remplaça la chaire de Science de l'éducation. Cependant la Sociologie ne disparut pas puisqu'on créera pour Paul Fauconnet ${ }^{39}$ (1874-1939), une maîtrise de conférence en Science de l'éducation et sociologie. Il devient, en 1932, titulaire de la chaire de Sociologie, qui était, à l'origine, une chaire de Langue et littérature anglaises. De plus, lorsque Célestin Bouglé devient, en 1935, directeur de l'ENS, Maurice Halbwachs (18771945) qui enseigna, à la fin de sa vie, la Sociologie au Collège de France, le remplaça à la chaire d'Histoire et d'économie sociale au poste de chargé de cours. Il occupera, en 1939, la chaire de Sociologie laissée vacante suite au décès de Paul Fauconnet.

Bien que d'autres facteurs entrent en ligne de compte, l'intérêt de la Sociologie pour l'étude des migrations est à inscrire dans ce contexte de développement.

Au cours des années 1960, on note une forte augmentation des étudiants inscrits dans les facultés de Lettres et « à l'intérieur des facultés de Lettres, les étudiants [...] s'inscrivent en masse dans les départements de sociologie et de psychologie [ ] » (Bénéton et Touchard, 1970 : 511). L'hypothèse selon laquelle l'augmentation du nombre des thèses en général et celle du nombre des thèses sur les migrations en particulier serait due aux effectifs croissants des étudiants inscrits peut être émise.

$\mathrm{Au}$ milieu de ces années, les thèses soutenues en Sociologie, furent davantage orientées vers les migrations et ceci sous l'influence des luttes anticolonialistes et de mai 68. On étudiait les aspirations culturelles des immigrants espagnols, la vie sociale des étudiants étrangers à Toulouse, les processus des migrations internes et externes et leur impact sur la croissance urbaine et le développement des activités tertiaires.

À partir des années 1970, le nombre des thèses sur les migrations n'a cessé d'augmenter : soixante-sept thèses entre 1970-1979, quatre-vingt-cinq pendant les années 1980 et quatre-vingt-quatorze pendant les années 1990. Selon Gérard Noiriel (1988), les causes de l'absence d'étude des migrations avant ces années sont à trouver dans la tradition non interventionniste des chercheurs ${ }^{40}$ dans les questions concernant les étrangers. De plus, le travail d'enquête sur le terrain, que nécessite généralement la recherche sur l'immigration, n'a pas été un facteur d'incitation auprès des sociologues formés dans les grandes institutions telle que l'ENS. L'immigration, en tant qu'objet d'étude, n'est pas valorisée au sein d'institutions bénéficiant d'un certain prestige. Sur ce point, Abdelmalek Sayad (1933-1998) a montré dans de nombreux travaux que « la dignité intellectuelle des objets sociaux est à la mesure de la dignité sociale de ces mêmes objets qu'on a convertis en objets intellectuels » (Sayad, 1991 : 20). Or, l'immigration, qui concerne une population dominée socialement et politiquement, est demeurée pendant longtemps, pour l'Université française, un objet de peu d'intérêt. Ainsi Louis Chevalier (1911-2001), qui exprima dans de nombreux articles le projet de recherches historiques sur l'immigration contemporaine, se détourna de cette ambition pour étudier l'histoire des migrations au XIXe siècle.

38 Sociologue, disciple d'Émile Durkheim. Agrégé de philosophie et directeur du Centre de documentation sociale à l'ENS, fondé grâce aux donations du riche banquier Albert Kahn (1860-1940).

39 Agrégé de philosophie. Il fut soutenu entre autres par Émile Durkheim pour être nommé maître de conférences à Paris.

40 Ceux de l'«école française » de sociologie et à leur tête Émile Durkheim. 
En 1962, il s'en est expliqué dans la préface du livre de Charlotte Roland : " par tradition, les disciplines [...] ne vont pas dans ce sens [de l'étude des immigrés]. La Sociologie, prioritaire en l'espèce bien au-dessus de ces contingences, s'en remettant, de ce soin, aux derniers échelons de sa hiérarchie : d'autant moins soucieuse d'ailleurs de s'y intéresser de nos jours que la sociologie américaine elle-même s'oriente désormais vers d'autres sujets, sursaturée d'études de cette espèce, s'estimant assez renseignée sur tel ou tel aspect du comportement professionnel, politique ou sexuel de l'Italien, du Polonais, du Noir ou du Chinois du coin, et abandonnant en bien fâcheuse posture ses fidèles imitateurs de France et d'ailleurs » (Roland, $1962: 8$ ).

Pendant cette décennie, l'immigré a obtenu d'autres statuts sans cesser d'avoir celui de travailleur : il est père de famille et à ce titre sa descendance est étudiée sous l'angle de son adaptation, de sa scolarisation, etc. On étudie aussi ses névroses et ses problèmes d'acculturation. Abdelmalek Sayad rappelle que la société d'accueil "ne se pose le problème des "immigrés" que pour autant que les immigrés lui "posent des problèmes” » (Sayad, $1991: 11)$.

Avec soixante-sept thèses soutenues en Sociologie entre 1970 et 1979, cette discipline montre une grande variété dans les sujets d'étude et dans les populations étudiées. En examinant les thèses, certaines auraient pu être soutenues en Sciences économiques ou en Démographie, mais ces disciplines semblent avoir réduit le champ de leurs intérêts. Le vide qu'elles laissèrent est comblé par la Sociologie qui est une science sociale générale. Son caractère hétéroclite peut être d'ordre institutionnel : alors que d'autres disciplines comme la Démographie disposaient d'instruments d'observation puissants, la Sociologie pour sa part s'est développée presque exclusivement au sein des universités. Par conséquent, les enquêtes sociologiques et les observations sont souvent « locales, de faible dimension et uniques dans le temps, orientant ainsi la discipline dans son ensemble vers la description d'objets concrets » (Boudon, 2002 : 226). En effet, les thèses analysent les migrations internes en France, de petits groupes, bien localisés ${ }^{41}$, ou les migrations de populations présentes en France ${ }^{42}$. Une majorité de thèses est consacrée à cet axe où l'on constate que certaines populations (les Algériens, par exemple), par l'ancienneté de leur migration, ont suscité plus de thèses que d'autres et avec des problématiques différentes ${ }^{43}$.

Les migrations de populations dans d'autres pays sont étudiées à travers l'examen des migrations internes ${ }^{44}$ ou internationales ${ }^{45}$, de groupes restreints ${ }^{46}$ ou de groupes plus $\operatorname{larges}^{47}$.

Par ailleurs, quatre-vingt-cinq thèses sont soutenues durant les années 1980 (puis quatre-vingt-quatorze pendant les années 1990), dont la majoritét8 ${ }^{48}$ porte sur les populations

41 Agriculteurs, exploitations agricoles familiales.

42 Espagnols, Soninkés, Martiniquais, Sarakolés, Portugais.

43 Famille, scolarisation des enfants, alphabétisation, acculturation, insertion socioprofessionnelle, logement.

44 Au Liban, Maroc, Bangladesh.

45 L'émigration chinoise au Pérou, l'exode des compétences en Iran.

46 Peul du Fuuta, Serers Nduts.

47 Guinéens à Dakar, migrants au Sénégal.

48 Deux thèses seulement ont été soutenues sur les migrations de Français.

REMI 2011 (27) 3 pp. 7-30 
immigrées en France. Dès le début des années 1980, l'intégration sociale des travailleurs immigrés, leur mobilité sociale et professionnelle, leurs conditions de vie et de travail sont étudiées. La Sociologie réitère son intérêt pour les difficultés scolaires des enfants des travailleurs immigrés algériens et s'interroge sur les mobilisations familiales et les conditions de scolarisation de ces enfants. De plus, elle analyse les aspects socioculturels de la communication chez les « jeunes immigrés algériens ». D'autres populations ont intéressé la recherche au cours de ces années ; les travailleurs turcs et marocains et leurs familles, les femmes «maghrébines » ou encore les « jeunes immigrés » et l'action de la justice par rapport à leur déviance, leurs stratégies identitaires ${ }^{49}$ et les réfugiés.

On s'intéresse, comme en Médecine, à la représentation de la maladie chez les travailleurs marocains, à la représentation de la santé et aux médecines traditionnelles, mais aussi à la pratique associative et à l'intégration sociale des familles nord-africaines, des Zaïrois, etc. On étudie des sujets directement liés à l'actualité de ces années : la polygamie et l'excision dans l'immigration africaine. Les thèses qui ont concerné les enfants d'immigrés « maghrébins » ${ }^{50}$, et qui avaient déjà mobilisé la recherche sociologique, sont nombreuses. Les migrations de populations (les Dogons) dans d'autres pays sont également étudiées.

De plus, pendant ces années, la problématique du retour des immigrés apparaît et fait même irruption ${ }^{51}$ dans la recherche française. Elle a concerné de nouvelles populations $^{52}$. Rappelons qu'à partir de 1978, suite à la crise économique, et dans le but de rétablir l'équilibre du marché de l'emploi et celui des échanges extérieurs, le gouvernement français a envisagé le départ d'un grand nombre d'immigrés.

\section{La Géographie}

La Géographie fut la première discipline parmi les Sciences sociales à être introduite dans l'Université française ${ }^{53}$. Elle s'est imposée à la faculté des Lettres à la fin de $1870^{54}$, car la guerre contre l'Allemagne nécessitait d'acquérir une bonne connaissance de l'espace européen, d'où la généralisation de l'enseignement de la Géographie. De plus, certains professeurs de géographie ont mis en évidence la capacité de cette discipline à servir les intérêts commerciaux et coloniaux de la France puisqu'elle permettait la découverte de sources de matières premières et de nouveaux marchés. Malgré cette popularité, et bien que l'École française de géographie ait été créée, sous l'impulsion de Paul Vidal de

49 Chinois, Japonais, Camerounais, Comoriens, en Corse, travailleurs sociaux d'origine étrangère, postiers antillais. Les femmes immigrées algériennes ont fait l'objet de plusieurs thèses qui ont concerné leurs stratégies d'émigration, leurs croyances et leurs valeurs, leur histoire migratoire et les changements familiaux et matrimoniaux les concernant.

50 Étude de leur scolarité et de la réussite scolaire particulièrement chez les filles, leurs référents identitaires, leur environnement, le mariage et le concubinage européano-maghrébins, les nouvelles stratégies participatives à travers les pratiques radiophoniques, l'Islam et la quête d'une identité culturelle, le militantisme.

51 Étudiée chez les Tunisiens, Marocains ou Algériens.

52 Ivoiriens, réfugiés chiliens.

53 Actuellement, la Géographie fait partie du Département des Sciences humaines et humanités au sein du groupe « Sciences du temps et de l'espace » (ministère délégué à la Recherche, 2009).

54 Avant 1870, une seule chaire lui était consacrée, à Paris. 
la Blache (1845-1918) à la fin du XIXe et au début du XXe siècle, la Géographie n'était pas encore autonome, mais son entrée à la faculté des Lettres ${ }^{55}$ lui assura sa différenciation ultérieure et donc son avenir.

Au cours des années 1970, de nouvelles problématiques liées aux populations immigrées sont étudiées ${ }^{56}$. Soixante-cinq thèses sont soutenues entre 1980 et 1989 (quatrevingt-huit entre 1990 et 1999) avec une dominante des recherches menées sur d'autres pays que la France, pendant les années 1980 comme pendant les années 1990. De plus, au cours des années 1990, l'intérêt pour les migrations de loisirs réapparaît, mais cette fois-ci, il concerne le $\mathrm{Maroc}^{57}$ et le Brésil ${ }^{58}$ où des pratiques de tourisme national sont analysées dans leurs dimensions sociale et géographique.

L'immigration en France est analysée à travers les questions de logement et d'intégration spatiale des immigrés ${ }^{59}$, des effets du regroupement familial et de l'examen de nouvelles populations ${ }^{60}$. Une géographie territoriale et locale s'est illustrée dans plusieurs thèses consacrées aux migrations internes en France ${ }^{61}$.

\section{L'Histoire}

En 1969, la recherche historique, pour la première fois, enregistre officiellement deux thèses ${ }^{62}$. Cette absence antérieure de l'étude des migrations en Histoire nous ramène aux questionnements et aux mises en garde d'Ernest Renan (1823-1892) lors de sa conférence "Qu'est-ce qu'une nation ? », à la Sorbonne le 11 mars 1882 : "L'oubli, et je dirai même l'erreur historique, sont un facteur essentiel de la création d'une nation, et c'est ainsi que le progrès des études historiques est souvent pour la nationalité un danger. L'investigation historique, en effet, remet en lumière les faits de violence qui se sont passés à l'origine de toutes les formations politiques, même de celles dont les conséquences ont été bienfaisantes » (Forest, 1991 : 34). Ainsi Ernest Renan, en réfutant un ensemble de critères (la race, la langue, la religion, la géographie et l'intérêt économique), pour définir une nation, s'inscrit dans la même lignée de pensée que Jules Michelet (1798-1874) lorsqu'il écrivait dans Le Peuple en 1848 « La France a fait la France, et l'élément fatal

55 La Géographie (chaires, cours, conférences ou maîtrises de conférences) est présente, en 1892, dans treize facultés de Lettres parmi les quinze qui existaient alors (Weisz, $1970: 85$ ).

56 Place de la main-d'œuvre étrangère dans la Communauté économique européenne, main-d'œuvre yougoslave, impact de l'immigration libanaise sur la vie du Liban, causes et conséquences de l'émigration espagnole.

57 M. Berriane, Tourisme national et migrations de loisirs au Maroc : étude géographique, Université de Tours, 1990.

58 C.-J. Moura de Castilho, Tourisme et mobilité socio-géographique des individus pauvres à Recife/Brésil, Université de Paris 3, 1999.

59 Risque du " ghetto » dans l'agglomération parisienne, conditions de logement et insertion résidentielle des étrangers, femmes immigrées dans les grands ensembles, développement de la première banlieue bordelaise.

60 Mauriciens, Indiens du Nord, Chinois, jeunes issus de l'immigration maghrébine.

61 P. Busuttil, À la recherche du développement : la Creuse, Université de Clermont-Ferrand 2, 1990 ; F. Morin, Évolution de l'espace péri-urbain de Saintes, Université de Bordeaux 3, 1990.

62 Elles sont consacrées aux réfugiés huguenots dans les Provinces-Unies (XVIIe siècle) et aux Américains à Nantes et à Saint-Nazaire (1917-1919). 
de race m'y semble secondaire » (Forest, 1991 : 45) ou de Fustel de Coulanges (18301889), qui répondait à l'historien allemand Théodore Mommsen (1817-1903) quand celui s'en est pris à la France lors de l'annexion de l'Alsace et de la Lorraine (1870) : «Ce qui distingue les nations, ce n'est ni la race, ni la langue. Les hommes sentent dans leur cœur qu'ils sont un même peuple lorsqu'ils ont une communauté d'idées, d'intérêts, d'affections, de souvenirs et d'espérances. Voilà ce qui fait la patrie » (de Coulanges, 1919 : 96). C'est, selon Gérard Noiriel (1988 : 27), cette relation qu'ont entretenue les historiens avec l'idée de la nation qui constitue l'un des éléments de compréhension du désintérêt de la recherche historique au sujet de l'immigration. Cependant, la conception de la nation propre à Ernest Renan n'empêcha pas d'autres historiens d'étudier la place des populations immigrées en France, à en juger par la nette progression des thèses soutenues - officiellement - à la fin du XXe siècle.

Il faudrait toutefois nuancer cette explication d'ordre idéologique, prendre en compte la particularité de l'objet d'étude et introduire des causes d'ordres institutionnel et structurel : contrairement aux sciences expérimentales comme l'écrivait Marc Bloch (1886-1944) en 1922, « les sciences sociales n'ont pas de laboratoire ; elles n'ont à leur disposition d'autres expériences que celles que leur offre naturellement le passé » (Bloch, 2006 : 326). Par ailleurs, toutes les disciplines ne disposaient pas de moyens financiers pour exister de manière officielle et autonome. De plus, on ne peut pas oublier les rivalités et les oppositions qui existaient entre les facultés de Droit et les facultés de Lettres. Bien qu'une chaire d'histoire contemporaine ait été mise sur pied en 1884, suivie trois ans plus tard, de celle d'histoire de la Révolution, l'Histoire ne pouvait pas encore prétendre à son autonomie. Rappelons que le cours d'histoire de l'économie sociale, fondé en 1893 à la Sorbonne, fut financé par le Comte de Chambrun. C'est ce manque de moyens qui explique peut-être le « retard » français en Sciences sociales, et plus particulièrement dans l'étude des migrations, contrairement aux universités allemandes qui disposaient de moyens importants.

Les douze thèses soutenues en Histoire, au cours des années 1970, examinent aussi bien l'émigration française que l'immigration en France et les migrations dans d'autres pays ${ }^{63}$. Pendant les années 1980, bien qu'on note une augmentation du nombre des thèses (28) sur ce sujet, l'Histoire demeure en retrait vis-à-vis de la Sociologie, des Sciences économiques, de la Géographie ou de la Médecine. Cependant, à partir des années 1990, on assiste à une importante augmentation des thèses soutenues (79).

L'intérêt porté par l'Histoire à l'étude de communautés particulières n'a cessé d'augmenter ${ }^{64}$ aussi bien en France qu'à l'étranger ${ }^{65}$. De nouveaux sujets de thèses qui auraient pu être traités aussi en Sociologie apparaissent et sont consacrés à l'Islam en France, à sa représentation dans la presse nationale française ou aux tensions entre les « générations musulmanes ». Mais ces sujets montrent l'émergence chez les histo-

63 Celles des Ewé du Sud-Togo, les immigrants dans un quartier de Barcelone, la diaspora chinoise dans l'océan Indien occidental et les émigrés balkaniques (Grecs, Dalmates, Albanais) à Venise, de 1204 à 1453.

64 Les « filles du roi », les « Queyrassins », les étrangers en milieu rural, les réfugiés politiques, les Russes, les mineurs polonais.

65 Italiens en Algérie, Alsaciens au Texas, Andalous au Maghreb. 
riens d'une nouvelle discipline : la Socio-histoire, à travers l'emprunt méthodologique et théorique à la Sociologie pour conduire leurs recherches en Histoire (Noiriel, 2001 : 480-484). Toutefois, au cours de cette décennie, on note l'éclosion d'approches historiques différentes inscrivant ainsi la permanence d'une séparation entre différents courants de la recherche historique : l'Histoire politique qui s'interroge sur le pouvoir colonial et les communautés étrangères à Madagascar ou sur l'Algérie, l'Histoire économique et sociale qui étudie la bourgeoisie blésoise ou les toucouleurs dans la région parisienne, l'Histoire régionale qui dresse le tableau des migrations dans l'Ain ou dans les Ardennes, par exemple.

\section{LA DÉMOGRAPHIE : UN CAS PARTICULIER}

Le Système universitaire de documentation (Sudoc) fait apparaître que les premières thèses soutenues en Démographie sur les migrations datent de la fin des années 1960. Ce fait est d'autant plus étonnant que l'on sait que la question des origines, et donc celle de l'immigration, a préoccupé des chercheurs comme Alphonse et Jacques Bertillon, Gustave Le Bon (1841-1931) ou Alfred Sauvy (1898-1990) dès le début du XXe siècle et bien d'autres chercheurs de la Fondation Alexis Carre ${ }^{66}$. Ainsi de nombreux travaux et statistiques sur la population étrangère ont été réalisés avec le soutien de l'État, à travers cette institution. Cependant, la première thèse en Démographie recensée ne date que de 1969 et porte sur les méthodes de surveillance de la santé des personnes travaillant hors de leur continent d'origine. Au cours des années qui suivirent, les thèses soutenues furent en légère augmentation (cinq en 1970-1979, huit en 1980-1989 et neuf en 1990-1999) et portèrent en majorité sur les migrations dans d'autres pays ${ }^{67}$. Les thèses dont le terrain de recherche se situait en France ont concerné principalement l'analyse des migrations internes, alternantes, la natalité et la fécondité des émigrées algériennes, la démographie de la communauté libanaise installée dans la région parisienne ou encore l'asile en France.

Il semble évident que le total des thèses soutenues officiellement en Démographie entre 1969 et 1999 (23) ne reflète aucunement l'intérêt de cette discipline au sujet des migrations, compte tenu du dynamisme de la Démographie historique qui faisait partie de l'Histoire sociale et dont le succès a été « rendu possible par une configuration institutionnelle, politique et scientifique propre aux années 1950 »(Rosental, 2003 : 129). En effet, lorsqu'on examine systématiquement la base de données des 1882 thèses, on remarque que plusieurs thèses, qui auraient pu être soutenues en Démographie, se sont développées en dehors de cette discipline ${ }^{68}$. Ce qui soulève la question plus large des effets des

66 Elle deviendra, en 1945, l'Institut national des études démographiques.

67 Maroc, Algérie, Niger, Rwanda, Syrie, Liban, Inde, Russie.

68 Les exemples sont multiples, j'en donne ici un bref aperçu : thèses soutenues en Géographie : E.

Arnould Bantquin, La population des espaces de faible densité en Lorraine. Étude démogéographique, Nancy 2, 1984 ; N. Kamel, Instruments démographiques et flux migratoires au Liban: critique et étude de cas : Maifadoun (Sud-Liban), Paris 4, 1985. En Histoire : D. Peter, Démographie et vie économique en Basse-Alsace : l'exemple de l'Outre-Forêt (1648-1848), Strasbourg 2, 1994. En Sociologie également comme dans d'autres disciplines, on trouve des thèses qui auraient pu être soutenues en Démographie (Benabou-Lucido, 2008 : annexe 9).

REMI 2011 (27) 3 pp. 7-30 
inscriptions disciplinaires qui ne correspondent pas à la réalité des approches théorique, méthodologique et thématique effectivement développées.

\section{FOISONNEMENT ET FRAGMENTATION DE L'ÉTUDE DES MIGRATIONS À PARTIR DES ANNÉES 1970}

Au cours de ces années, le nombre des thèses soutenues dans les anciennes disciplines (Géographie, Sociologie, etc.) n'a pas cessé d'augmenter. Parallèlement, de nouvelles disciplines ont fait des migrations leur objet d'étude ${ }^{69}$ et nous avons vu que certaines thèses étaient soutenues sous la tutelle de disciplines qui ne respectaient pas le découpage disciplinaire. Ainsi, dix-huit nouvelles disciplines introduisent les migrations dans leur champ d'étude, dans un contexte difficile lié à la division de l'Université française en treize universités nouvelles (Loi d'orientation d'Edgar Faure ${ }^{70}$ ).

L'on pourrait se réjouir de cet intérêt pour les migrations. Cependant, la multiplication des nouvelles perspectives de l'étude des migrations révèle, essentiellement, un caractère fragmenté : chaque discipline est cantonnée dans son champ de compétences ainsi que dans une aire géographique bien précise et traite les migrations à travers sa seule perspective. Le dialogue entre disciplines est quasiment absent. Tout au plus, se manifeste-t-il à travers les emprunts méthodologiques. Ainsi des méthodes d'analyse qu'on peut inscrire tantôt en Lettres, tantôt en Sciences humaines et sociales ${ }^{71}$ sont utilisées par certaines disciplines nouvelles. Par exemple, les Études anglaises, nord-américaines ${ }^{72}$, ibériques, latino-américaines, ou encore arabo-islamiques et germaniques, qui visent l'examen de la langue anglaise pour les premières, l'examen des langues portugaise, espagnole et arabe pour les secondes, ont recours aussi bien à la linguistique qu'à la littérature et à la civilisation pour traiter, entre autres, des migrations. L'étude linguistique de la langue devient une médiation, un moyen d'informations sur la relation qui existe entre le discours et la culture. Les thèses soutenues dans ces disciplines vont se développer au cours des années 1980. Ce développement va se poursuivre, pour certaines, pendant les années 1990 avec l'apparition des Études slaves et italiennes.

Dans cette même perspective, on peut intégrer les Littératures comparées dont l'objectif est l'analyse des œuvres d'écrivains exilés, ou la relation entre exil et création. De plus les disciplines Langues et littératures ${ }^{73}$ ou Littérature italienne ont étudié exclusivement la relation entre les migrations et la création littéraire, ou l'expérience de l'exil de certains écrivains.

69 Cf. annexe 2.

70 Loi du 12 novembre 1968, remplacée par la loi du 26 janvier 1984.

71 Études anglaises, latino-américaines, nord-américaines, ibériques, arabo-islamiques.

72 Notons que les études anglaises se trouvent généralement associées aux études nord-américaines.

73 Sémiologie, Littérature d'expression française, Littérature et expression, modernes et contemporaines option littérature, Littérature française, Sciences des textes et des documents, Littérature et civilisation comparées. 
De nouvelles disciplines se sont intéressées aussi aux migrations et ont contribué, bien qu'elles soient peu nombreuses, à l'augmentation du total général des thèses ${ }^{74}$. Mais, on ne peut pas dégager pour certaines leur orientation. L'on peut toutefois indiquer que le champ d'étude de ces disciplines conjugue les composantes migratoire et artistique.

Au cours des années 1990, d'autres disciplines viennent s'ajouter à cette liste : les Études grecques, la Littérature allemande, les Langues et cultures européennes, les Langues et la Littérature anglaise ${ }^{75}$. Dans ces disciplines, les migrations sont étudiées en empruntant des méthodes aussi bien sociologiques qu'historiques ${ }^{76}$.

De manière périphérique, d'autres disciplines se sont intéressées au thème des migrations : les Études urbaines liées directement au domaine de la Géographie de l'aménagement, les Sciences de gestion ${ }^{77}$ et le Cinéma. On trouve également des enquêtes épidémiologiques auprès des populations immigrées dans des disciplines scientifiques : en Chirurgie dentaire, en Pharmacie pour expliquer certaines pathologies de ces populations. Dans cette même catégorie, on constate l'apparition de thèses en : Mathématiques, Informatique et Sciences sociales ${ }^{78}$ et en Terre, Océan, Espace ${ }^{79}$.

Enfin, une thèse est soutenue en Sciences et techniques des activités physiques et sportives et porte sur l'insertion par le sport des jeunes d'origine « maghrébine », dans des banlieues en difficulté.

\section{CONCLUSION}

Comme nous l'avons montré à travers l'histoire de la constitution des disciplines et l'intérêt plus ou moins important de certaines d'entre elles à l'étude des migrations, la juxtaposition des écoles et des disciplines constatée n'est pas synonyme d'un traitement global des migrations. Quelques thèses seulement ont initié le dialogue entre les disciplines et certaines ont été soutenues à l'époque dans une discipline faute d'une séparation claire ou existante entre elles.

Ce travail met en évidence un cloisonnement important entre disciplines pour un sujet qui nécessite d'être traité dans sa globalité. Il montre l'importance aussi bien de la contextualisation de la recherche sur ces thématiques que les conséquences de l'organisation disciplinaire à l'Université d'un point de vue social, économique et politique. Ici, le problème lié à l'ancrage institutionnel des disciplines est déterminant et peut expliquer cette frilosité dans les échanges.

74 En Philosophie, Arts, Art et archéologie, Théâtre.

75 Cette discipline est à différencier des études anglaises déjà mentionnées. Elle a traité de $L a$ migration des communautés britanniques en France et en Espagne.

76 Études hispaniques, extrême-orientales, hébraïques, romanes.

77 Les thèses soutenues en Sciences de gestion portent exclusivement sur la population des cadres expatriés. Les problématiques étudiées se trouvent également traitées par les Sciences économiques.

78 Reconstruction et la dynamique des populations du passé.

79 Cette discipline dépend de l'Unité Histoire et civilisations : histoire et archéologie des mondes anciens et médiévaux, de l'art. 
Pourtant déjà, en 1890, Louis Liard parlait d'échanges et de dialogue entre les facultés : « dans l'Université, les Facultés, tout en restant individuelles, ne sont plus des compartiments étanches et impénétrables. Comme ceux des fruits cloisonnés, ces compartiments distincts ont des parois communes et perméables, et tous s'ouvrent sur le même cœur. Aussi est-ce de l'un et à l'autre un échange perpétuel, une exosmose et une endosmose incessantes. Tout ce qui se passe dans l'un retentit dans les autres ; rien de ce qui surgit de nouveau dans l'un n'est perdu pour les autres. Ainsi agencées, toutes les parties réagissent les unes sur les autres » (Liard, 1890 : 146-147).

Quelques années plus tard, Abel Châtelain attirait l'attention des chercheurs de différentes disciplines sur les méthodes employées pour l'explication des causes et des facteurs des migrations qui lui semblaient alors « discutables, très incomplètes et imparfaites ». Prenant l'exemple des démographes économistes, il nous informe qu'ils " n'ont souvent voulu mettre en relief que les causes démographiques et économiques : forte natalité conduisant à un surpeuplement, fortes densités humaines dépassant les possibilités économiques, nécessité de cette soupape de sûreté qu'est l'émigration, temporaire ou définitive. Les migrations humaines ne seraient donc qu'un phénomène d'équilibre démographique, en rapport avec les ressources, entre régions ou pays. Sans doute, les facteurs démographiques et économiques tiennent une très grande place. Mais on ne peut négliger les facteurs psychologiques et sociologiques, méconnaître les réactions et les comportements des masses devant les données économiques. Il y a une géographie sociologique des migrations qui n'a guère été abordée : selon les sexes, les milieux sociaux, les régions et les civilisations ; les comportements des individus ne sont pas toujours les mêmes quand il s'agit de quitter un pays d'origine » (Châtelain, 1963, 1-17).

L'approche pluridisciplinaire, malgré les avertissements de Louis Liard, ceux d'Abel Châtelain ou ceux encore d'Abdelmalek Sayad et les efforts de Gérard Noiriel pour la promouvoir se met en œuvre difficilement. Les disciplines restent cloisonnées, isolées et ne "voient » que le fragment qui est le leur, dans l'étude des migrations. Il semble qu'elles ne peuvent recevoir autre chose que ce qui peut contribuer à leurs objets propres, d'où leur spécialisation.

Certes, les migrations appartiennent aux champs d'étude d'une majorité de disciplines en France, mais du fait de cette appartenance multiple, elles ne relèvent d'aucunes.

Il semble que le cloisonnement des disciplines que j'ai tenté de mettre en évidence à partir des résumés de thèses est à mettre en parallèle avec celui des centres de recherche et des facultés, car le développement de l'étude des migrations est à analyser dans le cadre de cette division. Tout au long de cette analyse, on a vu combien le sujet des migrations a été morcelé, ce qui l'a empêché de devenir l'objet d'un savoir global. Afin d'y remédier, il faudrait faire apparaître les diverses interactions entre les migrations et la société d'accueil, la société d'origine ${ }^{80}$ et cela par le biais d'une analyse transversale et globale, menant à une compréhension convenable de ces migrations en tant que fait social contemporain.

80 Sur les 1882 thèses, une seule thèse s'est intéressée conjointement aux processus d'immigration et d'émigration. 
Tout ceci revient à inscrire la migration dans la société tout en prenant «ses distances avec "le présent" pour mieux le servir » (Noiriel, $1994: 123$ ) et sans omettre de retracer son histoire ou ses histoires, car « en bien des cas la connaissance du passé peut, non seulement, éclairer le présent, mais servir les hommes dans leurs actions » (Noiriel, 1994 : 124). Il faudrait parvenir à faire des migrations un objet d'étude global ou mieux encore un «fait social total ». Il reste une discipline à construire et à structurer où les approches mises en œuvre par les disciplines préexistantes seront conjuguées pour atteindre une meilleure appréhension des migrations même si cela suppose des efforts énormes de communication et de collaboration entre les disciplines qui disposent chacune d'une « esthétique de langage » (Bloch, 2006 : 867) qui leur est propre.

Cela revient à envisager l'interdisciplinarité pour l'étude des migrations selon les deux axes soulignés par Marc Bloch : l'emprunt de méthodes entre disciplines et la collaboration entre spécialistes de différentes disciplines afin de comprendre des problèmes définis en commun selon des critères bien établis. Dans cette nécessité de dialogue entre disciplines pour l'étude des migrations en tant que problématique sociale et contemporaine, il s'agit d'aller au-delà de l'appropriation des découvertes d'une discipline par une autre, il s'agit davantage d'une communication entre spécialistes et d'une réelle communauté professionnelle pour établir une nécessaire relation d'échange et des passerelles entre les disciplines de l'Université française (sciences sociales et humaines, médecine et santé, sciences et technologies de l'information et de la communication, etc.).

\section{Références bibliographiques}

BENABOU-LUCIDO Latifa (2008) Représentations sociales des migrations et rapports entre émigration et immigration : exemple des migrations marocaines, ss la dir. de Nancy L. Green, EHESS.

BÉNÉTON Philippe et TOUCHARD Jean (1970) Les interprétations de la crise de mai-juin 1968, Revue française de science politique, 20 (3), pp. 503-544.

BLOCH Marc (2006) L'histoire, la Guerre, la Résistance, Paris, Gallimard, 1176 p.

BOUDON Raymond (2002) Sociologie. A. Les développements, in Encyclopaedia Universalis, 21, Éditeur à Paris.

BOUDON Raymond, BESNARD Philippe, CHERKAOUI Mohamed et LÉCUYER Bernard-Pierre (Dir.) (1997) Dictionnaire de la sociologie, Paris, Larousse, 237 p.

CHÂTELAIN Abel (1963) Problèmes de méthodes. Les migrations de la population, Revue économique, 14 (1), pp. 1-17.

COLLARD Claude (Dir.) (2006) Des sources pour l'histoire de l'immigration en France de 1830 à nos jours : guide, Paris, BNF, $427 \mathrm{p}$.

COULANGES Fustel (de) (1919) Questions contemporaines, Paris, Librairie Hachette, (3ème éd.), $111 \mathrm{p}$.

DURKHEIM Émile (2004) Les règles de la méthode sociologique, Paris, PUF, (12ème éd.), 149 p.

FOREST Philippe (1991), Textes de Barrès, Daudet, Gourmont, Céline, Paris, Pierre Bordas et fils éditeur, $126 \mathrm{p}$.

GREEN Nancy L. (2005) The politics of exit: reversing the immigration paradigm, The journal of modern history, 77 (2), pp. 263-289.

GREEN Nancy L. (2002) Repenser les migrations, Paris, PUF, 138 p.

REMI 2011 (27) 3 pp. 7-30 
GREEN Nancy L. (1991) L'immigration en France et aux États-Unis. Historiographie comparée, Vingtième siècle. Revue d'histoire, 29 (29), pp. 67-82.

GREEN Nancy L. et WEIL François (Dir.) (2006) Citoyenneté et émigration : Les politiques du départ, Paris, EHESS, 274 p.

LIARD Louis (1909) L'Université de Paris. La vieille université - La nouvelle université - La nouvelle Sorbonne, Paris, H. Laurens Éditeur, 133 p.

LIARD Louis (1890) Universités et Facultés, Paris, Armand Colin, 229 p.

LIAUZU Claude (1999) L'obsession des origines : démographie et histoire des migrations, Mots, 60 (1), pp. 155-165.

LIAUZU Claude (Dir.) (1990) Si les immigrés m'étaient comptés, Paris, Syros, 238 p.

Marcel Jean-Christophe (2001) Georges Gurvitch : les raisons d'un succès, Cahiers internationaux de sociologie, 1 (110), pp. 97-119.

LIAUZU Claude (1982) Aux origines des tiers-mondismes : colonisés et anticolonialistes en France : 1919-1939, Paris, L'Harmattan, 274 p.

MAZON Brigitte (1988) Aux origines de l'E.H.E.S.S. Le rôle du mécénat américain, Paris, Le Cerf, $187 \mathrm{p}$.

NOIRIEL Gérard (2006) Introduction à la socio-histoire, Paris, La Découverte, $121 \mathrm{p}$.

NOIRIEL Gérard (2001) État, nation et immigration : vers une histoire du pouvoir, Paris, Gallimard, $399 \mathrm{p}$.

NOIRIEL Gérard (1994) En mémoire de Marc Bloch. Retour sur l'Apologie pour l'histoire, Genèses, 17 (1), pp. 122-139.

NOIRIEL Gérard (1988) Le creuset français. Histoire de l'immigration XIXe-XXe siècles, Paris, Le Seuil, $437 \mathrm{p}$.

PLAISANCE Éric et VERGNAUD Gérard (2005) Les sciences de l'éducation, Paris, La Découverte (1ère éd. 2001), 123 p.

PROST Antoine (1989) 1968 : mort et naissance de l'université française, XXe siècle, 23 (23), pp. 59-70.

ROLAND Charlotte (1962) Du ghetto à l'Occident. Deux générations yiddiches en France, Paris, Éditions de Minuit, 296 p.

REISSE Jacques (1999) L'interdisciplinarité, conséquence d'une division arbitraire du savoir, Revue de Géographie Alpine, 87 (1), pp. 13-18.

ROSENTAL Pierre-André (2003) La nouveauté d'un genre ancien : Louis Henry et la fondation de la démographie historique, Population, 58 (1), pp. 103-136.

SAYAD Abdelmalek (2001) La vacance comme pathologie de la condition d'immigré. Le cas de la retraite et de la pré-retraite, Revue Européenne des Migrations Internationales, 17 (1), pp. 11-36.

SAYAD Abdelmalek (1999) La double absence. Des illusions de l'émigré aux souffrances de l'immigré, Paris, Le Seuil, 438 p.

SAYAD Abdelmalek (1991) L'immigration ou les paradoxes de l'altérité, Paris, Éd. universitaires ; Bruxelles, De Boeck université, 331 p.

SAYAD Abdelmalek (1983) Le phénomène migratoire : une relation de domination, in Maghrébins en France : émigrés ou immigrés ?, Paris, CNRS, 426 p.

SAYAD Abdelmalek (1975) Elghorba : le mécanisme de reproduction de l'émigration, Actes de la recherche en sciences sociales, 1 (2), pp. 50-66.

SOUBIRAN-PAILLET Francine (2000) Juristes et sociologues français d'après-guerre : une rencontre sans lendemain, Genèses, 41, pp. 125-142.

WEISZ George (1979) L'idéologie républicaine et les sciences sociales. Les durkheimiens et la chaire d'histoire d'économie sociale à la Sorbonne, Revue française de sociologie, XX, pp. 83-112. 


\section{Annexes}

\section{Annexe 1 : Constitution de la base de données des thèses sur les migrations (BDDM)}

\section{Ressources}

Bibliographie de la France

Listes et bases de données des universités françaises et des centres de recherches

Le Système universitaire de documentation (Sudoc) qui présente :

1 Des avantages:

* Centralisation de toutes les thèses depuis le début des années 1800 , voire avant, soutenues en France ou à l'étranger, dans toutes les disciplines de la recherche

* À partir des années 1980, ces thèses sont accompagnées, le plus souvent, de résumés

2 Des faiblesses dues au fait que le Sudoc soit une base de données en réseau accueillant plusieurs sources de données, ce qui implique :

* Thèses en double ou en triple

* Erreurs concernant les noms des auteurs (donc plusieurs notices pour la même thèse)

* Erreurs sur les disciplines dans lesquelles les thèses ont été soutenues

* Notices incomplètes : pas de nom de l'auteur, pas de noms des directeurs de thèses jusqu'aux années 1980, absence de discipline ou de la date de soutenance

Interrogation de ces ressources à partir d'une liste de quarante-cinq mots-clés utilisés au singulier et au pluriel

Résultat : 13580 thèses

« Nettoyage » de la BDDM :

Éliminer les thèses portant des titres qui ne concernent pas les migrations humaines

Éliminer les thèses en double ou en triple

Repérer les thèses portant des titres a priori sans aucun lien avec les migrations humaines, mais qui traitent du sujet

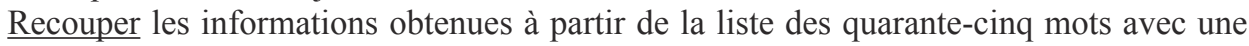
recherche en fonction des directeurs de thèses

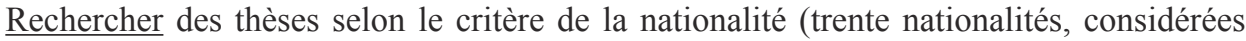
comme les plus représentatives du point de vue de leur présence en France $)^{81}$

81 Utilisation du guide des sources de l'immigration publié par la BNF (Collard, 2006) et du recensement de l'Insee de 1999 (Immigrés selon le pays de naissance en 1999). 


\section{Annexe 2 : Répartition des thèses soutenues sur les migrations, entre 1815 et 1999, par discipline}

\begin{tabular}{|c|c|c|c|c|c|c|c|c|c|c|c|c|}
\hline Discipline & $\begin{array}{l}\stackrel{2}{\infty} \\
\frac{1}{n} \\
\frac{\infty}{0}\end{array}$ & 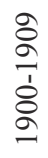 & $\begin{array}{l}\frac{a}{a} \\
\frac{1}{0} \\
\frac{a}{a}\end{array}$ & 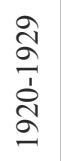 & 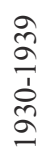 & $\begin{array}{l}\frac{9}{d} \\
\frac{1}{d} \\
\stackrel{+}{a}\end{array}$ & $\begin{array}{l}\frac{a}{2} \\
\frac{1}{2} \\
2\end{array}$ & 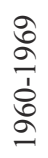 & 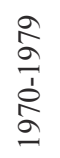 & $\begin{array}{l}\stackrel{2}{\circ} \\
\stackrel{\circ}{\circ} \\
\stackrel{\infty}{\circ}\end{array}$ & $\frac{2}{\frac{\sigma}{2}}$ & Total \\
\hline Droit-1815 & 66 & 39 & 23 & 36 & 36 & 15 & 10 & 4 & 11 & 24 & 44 & 308 \\
\hline Lettres-1883 & 1 & & & 1 & 9 & 1 & 3 & 9 & 9 & 8 & 16 & 57 \\
\hline Médecine-1908 & & 1 & & 1 & 2 & & & 1 & 5 & 185 & 93 & 288 \\
\hline $\begin{array}{l}\text { Sciences politiques et } \\
\text { économiques-1908 }\end{array}$ & & 1 & & 3 & & & & & & & & 4 \\
\hline Sciences économiques-1953 & & & & & & & 3 & 1 & 15 & 49 & 30 & 98 \\
\hline Géographie-1962 & & & & & & & & 4 & 31 & 65 & 88 & 188 \\
\hline Sociologie-1966 & & & & & & & & 5 & 67 & 85 & 94 & 251 \\
\hline Démographie-1969 & & & & & & & & 1 & 5 & 8 & 9 & 23 \\
\hline Histoire-1969 & & & & & & & & 2 & 12 & 28 & 79 & 121 \\
\hline Anthropologie-1971 & & & & & & & & & 10 & 17 & 25 & 52 \\
\hline Psychologie-1971 & & & & & & & & & 18 & 46 & 38 & 102 \\
\hline Études anglaises-1972 & & & & & & & & & 1 & 12 & 23 & 36 \\
\hline Linguistique-1973 & & & & & & & & & 4 & 16 & 19 & 39 \\
\hline Science politique-1973 & & & & & & & & & 3 & 9 & 18 & 30 \\
\hline $\begin{array}{l}\text { Études extrême- } \\
\text { orientales-1973 }\end{array}$ & & & & & & & & & 2 & 1 & & 3 \\
\hline Études orientales-1974 & & & & & & & & & 1 & & & 1 \\
\hline $\begin{array}{l}\text { Études latino- } \\
\text { américaines-1975 }\end{array}$ & & & & & & & & & 2 & 11 & 4 & 17 \\
\hline Littérature comparée-1975 & & & & & & & & & 2 & 1 & 2 & 5 \\
\hline Études ibériques-1976 & & & & & & & & & 3 & 6 & 8 & 17 \\
\hline $\begin{array}{l}\text { Études nord- } \\
\text { américaines-1976 }\end{array}$ & & & & & & & & & 4 & 9 & 17 & 30 \\
\hline Études urbaines-1976 & & & & & & & & & 1 & 3 & 2 & 6 \\
\hline $\begin{array}{l}\text { Sciences de l'information et } \\
\text { de la communication-1977 }\end{array}$ & & & & & & & & & 2 & 1 & 1 & 4 \\
\hline Sciences de gestion-1978 & & & & & & & & & 1 & & 3 & 4 \\
\hline Cinéma-1979 & & & & & & & & & 1 & & 2 & 3 \\
\hline $\begin{array}{l}\text { Études arabo- } \\
\text { islamiques-1979 }\end{array}$ & & & & & & & & & 1 & 7 & 3 & 11 \\
\hline Études germaniques-1979 & & & & & & & & & 1 & 10 & 9 & 20 \\
\hline Sciences de l'éducation-1979 & & & & & & & & & 4 & 30 & 34 & 68 \\
\hline Philosophie-1980 & & & & & & & & & & 1 & 2 & 3 \\
\hline Art et archéologie-1981 & & & & & & & & & & 1 & 1 & 2 \\
\hline Études africaines-1981 & & & & & & & & & & 3 & & 3 \\
\hline Études hispaniques-1981 & & & & & & & & & & 1 & & 1 \\
\hline Sémiologie-1981 & & & & & & & & & & 1 & & 1 \\
\hline
\end{tabular}




\begin{tabular}{|c|c|c|c|c|c|c|c|c|c|c|c|c|}
\hline Théâtre-1981 & & & & & & & & & & 1 & 1 & 2 \\
\hline Pharmacie-1983 & & & & & & & & & & 8 & 2 & 10 \\
\hline Chirurgie dentaire-1985 & & & & & & & & & & 5 & 1 & 6 \\
\hline $\begin{array}{l}\text { Littérature et expression } \\
\text { modernes et contemporaines } \\
\text { option littérature-1985 }\end{array}$ & & & & & & & & & & 1 & & 1 \\
\hline $\begin{array}{l}\text { Études extrême-orientales - } \\
1986\end{array}$ & & & & & & & & & & 1 & & 1 \\
\hline Littérature française-1986 & & & & & & & & & & 3 & 4 & 7 \\
\hline Études hébraïques-1987 & & & & & & & & & & 1 & 2 & 3 \\
\hline Études romanes-1987 & & & & & & & & & & 1 & 1 & 2 \\
\hline Littérature allemande-1987 & & & & & & & & & & 1 & 1 & 2 \\
\hline Littérature italienne-1987 & & & & & & & & & & & 5 & 5 \\
\hline $\begin{array}{l}\text { Sciences des textes et des } \\
\text { documents-1989 }\end{array}$ & & & & & & & & & & 1 & & 1 \\
\hline $\begin{array}{l}\text { Mathématique, Informatique } \\
\text { et sciences sociales-1991 }\end{array}$ & & & & & & & & & & & 1 & 1 \\
\hline Sciences du langage-1991 & & & & & & & & & & & 6 & 6 \\
\hline Études italiennes-1992 & & & & & & & & & & & 5 & 5 \\
\hline Études slaves-1992 & & & & & & & & & & & 5 & 5 \\
\hline Langues-1992 & & & & & & & & & & & 1 & 1 \\
\hline $\begin{array}{l}\text { Littérature et civilisation } \\
\text { comparées-1994 }\end{array}$ & & & & & & & & & & & 3 & 3 \\
\hline Terre, océan, espace-1995 & & & & & & & & & & & 1 & 1 \\
\hline Études grecques-1996 & & & & & & & & & & & 1 & 1 \\
\hline Littérature anglaise-1996 & & & & & & & & & & & 1 & 1 \\
\hline Musicologie-1996 & & & & & & & & & & & 2 & 2 \\
\hline Sciences de l'art-1997 & & & & & & & & & & & 1 & 1 \\
\hline $\begin{array}{l}\text { Langues et cultures } \\
\text { européennes-1998 }\end{array}$ & & & & & & & & & & & 1 & 1 \\
\hline Langage et parole-1999 & & & & & & & & & & & 1 & 1 \\
\hline $\begin{array}{l}\text { Langues, littérature, société- } \\
1999\end{array}$ & & & & & & & & & & & 1 & 1 \\
\hline $\begin{array}{l}\text { Sciences et techniques } \\
\text { des activités physiques et } \\
\text { sportives-1999 }\end{array}$ & & & & & & & & & & & 1 & 1 \\
\hline \multirow[t]{2}{*}{ Discipline non identifiée } & & & & & & & 1 & 2 & 1 & 3 & 8 & 15 \\
\hline & 67 & 41 & 23 & 41 & 47 & 16 & 17 & 29 & 217 & 664 & 720 & 1882 \\
\hline
\end{tabular}




\section{Annexe 3 : Les nouvelles disciplines apparues au cours des années 1970}

\begin{tabular}{|c|c|c|c|}
\hline \multirow[b]{2}{*}{ Discipline } & \multicolumn{3}{|c|}{$\begin{array}{c}\text { Nombre des thèses soutenues et particularités et/ou } \\
\text { problématiques }\end{array}$} \\
\hline & $1970-1979$ & 1980-1989 & 1990-1999 \\
\hline $\begin{array}{l}\text { Ethnologie et } \\
\text { Anthropologie sociale } \\
\text { et culturelle-1971 }\end{array}$ & \begin{tabular}{l}
\multicolumn{1}{c}{10} \\
Terrain de recherche \\
exclusivement dans \\
d'autres pays que la \\
France. \\
Étude des migrations \\
dans des sociétés \\
relativement \\
homogènes et de \\
petite échelle
\end{tabular} & \begin{tabular}{l}
\multicolumn{1}{c}{17} \\
Élargissement du \\
champ d'étude : \\
populations \\
immigrées en France \\
et à l'étranger
\end{tabular} & \begin{tabular}{l}
\multicolumn{1}{c}{25} \\
Tendance des années \\
1980 confirmée
\end{tabular} \\
\hline Psychologie-1971 & \begin{tabular}{l}
\multicolumn{1}{c}{18} \\
Étude des \\
migrations, \\
essentiellement du \\
point de vue des \\
problèmes
\end{tabular} & \begin{tabular}{l}
\multicolumn{1}{c}{46} \\
Étude du « problème \\
du retour » en \\
plus de différentes \\
pathologies des \\
immigrés
\end{tabular} & \begin{tabular}{l}
\multicolumn{1}{c}{38} \\
Mêmes orientations, \\
mais de nouvelles \\
problématiques \\
plus générales ou \\
qui concernent \\
des populations \\
particulières
\end{tabular} \\
\hline Linguistique-1973 & \begin{tabular}{l}
\multicolumn{1}{c}{4} \\
Étude de la \\
population des \\
« enfants immigrés » \\
par rapport à \\
l'acquisition du \\
langage et aux \\
problèmes qui lui \\
sont liés \\
\end{tabular} & \begin{tabular}{l}
\multicolumn{1}{c}{16} \\
Mêmes \\
préoccupations \\
des années 1970 et \\
étude du langage des \\
travailleurs immigrés
\end{tabular} & $\begin{array}{c}19 \\
\text { Mêmes orientations } \\
\text { que les années } 1980\end{array}$ \\
\hline $\begin{array}{l}\text { Science politique- } \\
1973\end{array}$ & \begin{tabular}{l}
\multicolumn{1}{c}{3} \\
La dimension \\
politique comme \\
facteur d'explication \\
du fait migratoire \\
\end{tabular} & Idem & Idem \\
\hline $\begin{array}{l}\text { Sciences de } \\
\text { l'information et de la } \\
\text { communication-1977 }\end{array}$ & \begin{tabular}{l}
\multicolumn{1}{c}{2} \\
Analyse des \\
problèmes \\
d'information posés \\
aux populations \\
immigrées
\end{tabular} & Idem & Idem \\
\hline $\begin{array}{l}\text { Sciences de } \\
\text { l'éducation-1979 }\end{array}$ & \begin{tabular}{l}
\multicolumn{1}{c}{4} \\
Problèmes \\
des enfants de \\
travailleurs immigrés \\
à l'école française
\end{tabular} & Idem & Idem \\
\hline
\end{tabular}




\title{
Histoire du développement de la recherche universitaire française sur les migrations internationales (1815-1999) \\ Latifa Benabou-Lucido
}

Dans le cadre de sa recherche doctorale, l'auteur a pu explorer le statut des migrations en tant qu'objet d'étude dans la recherche française. À partir de ses résultats, l'auteur démontre que l'étude des migrations, à l'université française, est disséminée entre les nombreuses disciplines de la recherche et qu'il existe une prolifération des perspectives et des méthodes pour leur traitement. La fragmentation de l'étude des migrations traduit celle de l'institution universitaire française.

\section{History of French Academic Researches' Development on International Migrations (1815-1999)}

\author{
Latifa Benabou-Lucido
}

\begin{abstract}
A chapter dedicated to the status of migrations, as a French research's object was my thesis' (2008) introduction. It pointed out that migration's studies, at the French university, are spread between the several academic disciplines and that there is a proliferation of perspectives and methods for their treatment. This work emphasized the migrations' study's fragmentation and, there even, that of the French university institution.
\end{abstract}

\section{Historia del desarrollo de la investigación de las universidades francesas de las migraciones internacionales (1815-1999) \\ Latifa Benabou-Lucido}

En el marco de mi tésis sobre las migraciones (2008), se elaboró un capítulo sobre el estado de las migraciones como tema de estudio en la invetigación francesa. Se determinó que el estudio de las migraciones en las universidades francesas está distribuido entre las numerosas disciplinas de la investigación, y que existen múltiples y variadas perspectivas y métodos para su tratamiento. Este capitulo se centró en la fragmentación del estudio de migraciones y de las instituciones universitarias francesas. 